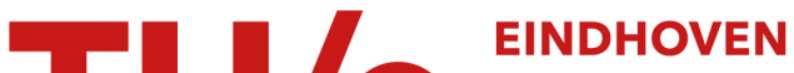 UNIVERSITY OF TECHNOLOGY
}

\section{Implementation of a MIMO OFDM-based wireless LAN system}

Citation for published version (APA):

Zelst, van, A., \& Schenk, T. C. W. (2004). Implementation of a MIMO OFDM-based wireless LAN system. IEEE Transactions on Signal Processing, 52(2), 483-494. https://doi.org/10.1109/TSP.2003.820989

DOI:

10.1109/TSP.2003.820989

Document status and date:

Published: 01/01/2004

\section{Document Version:}

Publisher's PDF, also known as Version of Record (includes final page, issue and volume numbers)

\section{Please check the document version of this publication:}

- A submitted manuscript is the version of the article upon submission and before peer-review. There can be important differences between the submitted version and the official published version of record. People interested in the research are advised to contact the author for the final version of the publication, or visit the $\mathrm{DOI}$ to the publisher's website.

- The final author version and the galley proof are versions of the publication after peer review.

- The final published version features the final layout of the paper including the volume, issue and page numbers.

Link to publication

\section{General rights}

Copyright and moral rights for the publications made accessible in the public portal are retained by the authors and/or other copyright owners and it is a condition of accessing publications that users recognise and abide by the legal requirements associated with these rights.

- Users may download and print one copy of any publication from the public portal for the purpose of private study or research.

- You may not further distribute the material or use it for any profit-making activity or commercial gain

- You may freely distribute the URL identifying the publication in the public portal.

If the publication is distributed under the terms of Article $25 f a$ of the Dutch Copyright Act, indicated by the "Taverne" license above, please follow below link for the End User Agreement:

www.tue.nl/taverne

Take down policy

If you believe that this document breaches copyright please contact us at:

openaccess@tue.nl

providing details and we will investigate your claim. 


\title{
Implementation of a MIMO OFDM-Based Wireless LAN System
}

\author{
Allert van Zelst, Student Member, IEEE, and Tim C. W. Schenk, Student Member, IEEE
}

\begin{abstract}
The combination of multiple-input multiple-output (MIMO) signal processing with orthogonal frequency division multiplexing (OFDM) is regarded as a promising solution for enhancing the data rates of next-generation wireless communication systems operating in frequency-selective fading environments. To realize this extension of OFDM with MIMO, a number of changes are required in the baseband signal processing. An overview is given of the necessary changes, including time and frequency synchronization, channel estimation, synchronization tracking, and MIMO detection. As a test case, the OFDM-based wireless local area network (WLAN) standard IEEE 802.11a is considered, but the results are applicable more generally. The complete MIMO OFDM processing is implemented in a system with three transmit and three receive antennas, and its performance is evaluated with both simulations and experimental test results. Results from measurements with this MIMO OFDM system in a typical office environment show, on average, a doubling of the system throughput, compared with a single antenna OFDM system. An average expected tripling of the throughput was most likely not achieved due to coupling between the transmitter and receiver branches.
\end{abstract}

Index Terms-MIMO systems, orthogonal frequency division multiplexing (OFDM), space division multiplexing, synchronization, wireless LAN.

\section{INTRODUCTION}

$\mathbf{T}$ HE MAIN goals in developing next-generation wireless communication systems are increasing the link throughput (bit rate) and the network capacity. Important improvements in throughput can be achieved when multiple antennas are applied at both the transmitter and receiver side, especially in a rich scattering environment. This has been shown for wireless communication links in both flat-fading [1] as well as frequency-selective fading channels [2].

Multiple-transmit multiple-receive antenna, i.e., multiple-input multiple-output (MIMO), techniques can basically be split into two groups: space time coding (STC) [3] and space

Manuscript received December 13, 2002; revised April 22, 2003. This work is sponsored by the Dutch cooperative research project B4 BroadBand Radio@Hand, BTS01063 and Agere Systems, The Netherlands. The main part of the research was carried out at the Wireless Systems Research Department, Agere Systems, Nieuwegein, The Netherlands. The associate editor coordinating the review of this paper and approving it for publication was Prof Dhananjay A. Gore.

The authors are with the Telecommunications Technology and Electromagnetics Group, Eindhoven University of Technology, Eindhoven, The Netherlands, and also with Agere Systems (formerly the Micro-Electronics Group of Lucent Technologies), Nieuwegein, The Netherlands. (e-mail: avanzelst@ieee.org; T.C.W.Schenk@tue.nl)

Digital Object Identifier 10.1109/TSP.2003.820989 division multiplexing (SDM) [1], [2], [4]. STC increases the performance of the communication system by coding over the different transmitter branches, whereas SDM achieves a higher throughput by transmitting independent data streams on the different transmit branches simultaneously and at the same carrier frequency. Since increasing the bit rates is our goal, we will focus on SDM algorithms in this paper.

A potential application of the MIMO principle is the nextgeneration wireless local area network (WLAN). The current WLAN standards IEEE 802.11a [5] and IEEE 802.11g [6] are based on orthogonal frequency division multiplexing (OFDM) [7]. A potential high data rate extension of these standards could be based on MIMO [8]. This leads to the promising combination of the data rate enhancement of SDM with the relatively high spectral efficiency and the robustness against frequency-selective fading and narrowband interference of OFDM. An advantage of wireless LAN systems is that they are mainly deployed in indoor environments. These environments are typically characterized by a richly scattered multipath. As explained in [1], this is a good condition for having a high MIMO capacity.

The goal of this paper is to prove the concept of increasing the link throughput by extending an OFDM-based system with MIMO, while focusing on the IEEE 802.11a standard. Although some publications present MIMO OFDM system design and measurements [9]-[11], a good overview of the required changes on synchronization algorithms when extending OFDM with MIMO, is, to our knowledge, still lacking in the literature. Since synchronization is an essential task for any digital communication system and required for reliable reception of the transmitted data, this paper provides an overview of the necessary changes to the preamble, time and frequency synchronization, channel estimation, and synchronization tracking. Furthermore, enhanced MIMO detection algorithms are proposed, based on per-antenna-coding (PAC) (sometimes called horizontal coding [12]) at the transmitter, i.e., PAC soft-output maximum likelihood detection (MLD) and PAC V-BLAST. Finally, to verify the proposed synchronization and detection algorithms, the bit error rate (BER) and packet error rate (PER) performance of the total implementation are evaluated both with simulations and initial measurements. The initial BER and PER measurements are performed for a number of locations in a typical office environment with a MIMO OFDM test system with three transmit and three receive antennas.

The layout of the paper is as follows. Section II describes the proposed MIMO OFDM transmitter and receiver scheme. In Section III, the MIMO OFDM signal model is defined. In 
Section IV, the implementation of the MIMO OFDM synchronization and detection algorithms are presented, and the performance is shown in Section V. Finally, conclusions are drawn in Section VI.

\section{SYSTEM DESCRIPTION}

Consider a MIMO OFDM system with $N_{t}$ transmit (TX) and $N_{r}$ receive (RX) antennas. When the MIMO technique of spatial multiplexing is applied [8], encoding can be done either jointly over the multiple transmitter branches or per branch [12]. The latter option is chosen as the encoding scheme in this paper and is called per-antenna-coding (PAC). A transmitter scheme in which PAC is applied to MIMO OFDM, is shown in Fig. 1. Basically, the MIMO OFDM transmitter consists of $N_{t}$ OFDM transmitters [13], among which the incoming bits are multiplexed, and then, each branch in parallel performs encoding, interleaving ( $\Pi$ ), QAM mapping, and $N_{c}$-point inverse discrete fourier transformation (IDFT) and adds a cyclic prefix (CP) before the final TX signal is upconverted to radio frequency (RF) and transmitted. For reliable detection, it is typically necessary that the receiver knows the wireless communication channel and keeps track of phase and amplitude drifts. To enable estimation of the wireless communication channel, the transmitter occasionally sends known training symbols. In WLANs, a preamble, which includes channel training sequences, is added to every packet. Moreover, to track the phase drift, pilot symbols are inserted into every MIMO OFDM data symbol on predefined subcarriers.

The receiver first must estimate and correct for the frequency offset and the symbol timing, e.g., by using the training symbols in the preamble. Subsequently, the CP is removed, and the $N_{c}$-point discrete fourier transformation (DFT) is performed per receiver branch. Since the MIMO algorithms that are proposed in this paper are single carrier algorithms, MIMO detection has to be done per OFDM subcarrier [8]. Therefore, the received signals of subcarrier $i$ are routed to the $i$ th MIMO detector to recover the $N_{t}$ data signals transmitted on that subcarrier. Next, the symbols per TX stream are combined, and finally, demapping, deinterleaving $\left(\Pi^{-1}\right)$, and decoding are performed for the $N_{t}$ parallel streams and the resulting data are combined to obtain the binary output data. Section IV-F explains how the detection and decoding block of Fig. 2 is filled in for PAC soft-decision output maximum likelihood detection (PAC SOMLD) and PAC vertical BLAST (PAC V-BLAST).

\section{MIMO OFDM SIGNAL MODEL}

Given the system description of Section II, we can develop a MIMO OFDM signal model. In this paper, we will need both a time-domain and frequency-domain model, i.e., we are looking for the relation between time-domain MIMO vectors $\mathbf{u}$ and $\mathbf{r}$ and the frequency-domain MIMO OFDM vectors $\hat{\mathbf{s}}$ and $\hat{\mathbf{x}}$ of Figs. 1 and 2, respectively.

Suppose that a communication system consists of $N_{t}$ TX and $N_{r}$ RX antennas and is denoted as a $N_{t} \times N_{r}$ system, where the transmitter at a discrete time instance $\tau$ sends an $N_{t}$-dimensional complex vector $\mathbf{u}(\tau)$, and the receiver records an $N_{r}$-dimensional complex vector $\mathbf{r}(\tau)$. For the simulations

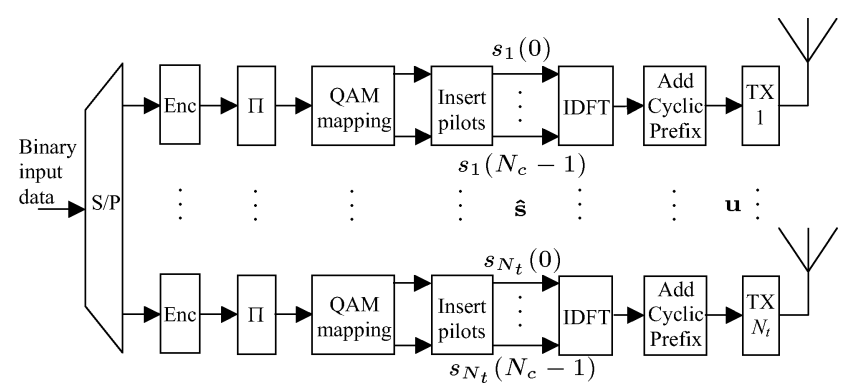

Fig. 1. Multiple-input multiple-output (MIMO) orthogonal frequency division multiplexing (OFDM) transmitter scheme.

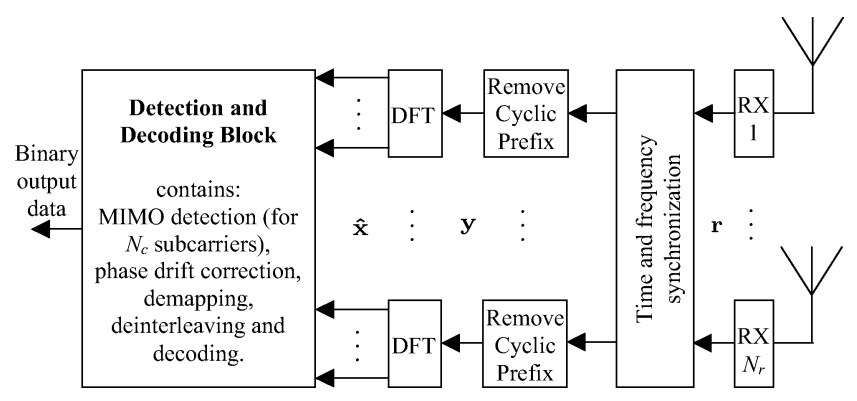

Fig. 2. MIMO OFDM receiver scheme.

in this paper, we assume that the system is operating in a frequency-selective Rayleigh fading environment and that the communication channel remains constant during a packet transmission, i.e., quasistatic fading. Suppose that the channel impulse response can be recorded with $L$ time instances, i.e., time samples, then the fading channel between the $p$ th TX and $q$ th RX antenna can be modeled by a discrete-time baseband equivalent $(L-1)$ th-order finite impulse response (FIR) filter with filter taps $g_{q p}(l)$, with $l=\{0, \ldots, L-1\}$. We assume that these taps are independent zero-mean complex Gaussian random variables with variance $\frac{1}{2} P(l)$ per dimension. The ensemble $P(l), l=\{0, \ldots, L-1\}$ is called the power delay profile (PDP), and its total power is assumed to be normalized to $\sigma_{c}^{2}=1$, which is the average channel or propagation attenuation. Suppose $g_{q p}(l)$ is the $(q, p)$ th element of the matrix $\mathbf{G}(l)$; then, the discrete-time MIMO baseband signal model is given by

$$
\mathbf{r}(\tau)=\sum_{l=0}^{L-1} \mathbf{G}(l) \mathbf{u}(\tau-l)+\mathbf{v}(\tau)
$$

where $\mathbf{v}(\tau)$ represents additive white Gaussian noise (AWGN) at the $\tau$ th sample with $N_{r}$ independent and identically distributed (i.i.d.) zero-mean, complex Gaussian elements with variance $(1 / 2) \sigma_{v}^{2}$ per dimension. The elements of $\mathbf{u}(\tau)$ are assumed zero mean, uncorrelated random variables with variance $\sigma_{u}^{2}$. Therefore, the expected SNR per receive antenna can be shown to be $\rho=N_{t} \sigma_{u}^{2} / \sigma_{v}^{2}$. Note that, for a fair comparison, we want to keep the total TX power the same as in the single-input single-output (SISO) case. Therefore, the power per TX antenna is scaled down by a factor $N_{t}$.

To deal with the frequency selectivity of the channel, we apply OFDM, utilizing $N_{c}$ subcarriers per antenna transmission. To combat inter-symbol interference (ISI), a CP of $N_{g}$ samples 
is added to the OFDM symbols. When $L \leq N_{g}+1$, ISI does not occur on a MIMO OFDM symbol basis, and the $a$ th received MIMO OFDM symbol (after removal of the $\mathrm{CP}$ ) is given by

$$
\hat{\mathbf{y}}(a)=\mathbf{C} \hat{\mathbf{u}}(a)+\hat{\mathbf{v}}(a), \quad a=0,1, \ldots
$$

where, with $N_{\text {tot }}=N_{g}+N_{c}$

$$
\begin{aligned}
& \hat{\mathbf{u}}(a)=\left(\begin{array}{c}
\mathbf{u}\left(a N_{\mathrm{tot}}+N_{g}\right) \\
\vdots \\
\mathbf{u}\left(a N_{\mathrm{tot}}+N_{\mathrm{tot}}-1\right)
\end{array}\right) \\
& \hat{\mathbf{v}}(a)=\left(\begin{array}{c}
\mathbf{v}\left(a N_{\mathrm{tot}}+N_{g}\right) \\
\vdots \\
\mathbf{v}\left(a N_{\mathrm{tot}}+N_{\mathrm{tot}}-1\right)
\end{array}\right)
\end{aligned}
$$

and $\mathrm{C}$ is an $N_{c} N_{r} \times N_{c} N_{t}$ block circulant matrix. In general, an $N A \times N B$ matrix is called block circulant if it has the form

$$
\mathbf{C}=\left(\begin{array}{ccccc}
\mathbf{C}_{0} & \mathbf{C}_{N-1} & \mathbf{C}_{N-2} & \ldots & \mathbf{C}_{1} \\
\mathbf{C}_{1} & \mathbf{C}_{0} & \mathbf{C}_{N-1} & \ldots & \mathbf{C}_{2} \\
\mathbf{C}_{2} & \mathbf{C}_{1} & \mathbf{C}_{0} & \ldots & \mathbf{C}_{3} \\
\vdots & \vdots & \vdots & \ddots & \vdots \\
\mathbf{C}_{N-1} & \mathbf{C}_{N-2} & \mathbf{C}_{N-3} & \ldots & \mathbf{C}_{0}
\end{array}\right)
$$

where the blocks, which are denoted by $\mathbf{C}_{x}$, are $A \times B$ matrices. Note that the block circulant matrix is fully defined by its first $N A \times B$ "block vector." In our case, this first "block of columns" is $N_{c} N_{r} \times N_{t}$ dimensional and given by

$$
\mathbf{K}=\left(\begin{array}{c}
\mathbf{G}(0) \\
\vdots \\
\mathbf{G}(L-1) \\
\mathbf{0}_{N_{r}\left(N_{c}-L\right) \times N_{t}}
\end{array}\right)
$$

where $\mathbf{0}_{N_{r}\left(N_{c}-L\right) \times N_{t}}$ is the $N_{r}\left(N_{c}-L\right) \times N_{t}$ all zeros matrix. When $\mathbf{s}(i, a)$ represents the MIMO vector transmitted on the $i$ th subcarrier of the $a$ th MIMO OFDM symbol and $s_{p}(i, a)$ is its $p$ th element, i.e., transmitted on the the $p$ th TX antenna, the transmitted block of samples in the time-domain becomes

$$
\hat{\mathbf{u}}(a)=\left(\mathbf{F}^{-1} \otimes \mathbf{I}_{N_{t}}\right)\left(\begin{array}{c}
\mathbf{s}(0, a) \\
\vdots \\
\mathbf{s}\left(N_{c}-1, a\right)
\end{array}\right)
$$

where $\otimes$ denotes the Kronecker product, $\mathbf{F}$ is the $N_{c} \times N_{c}$ Fourier matrix, of which the $(i, k)$ th element equals $\exp \left(-j 2 \pi\left(i k / N_{c}\right)\right)$, and $\mathbf{I}_{N}$ represents the $N \times N$-dimensional identity matrix. Finally, by taking the DFT at the receiver, we obtain the frequency-domain MIMO OFDM baseband signal model (under the assumption that $L \leq N_{g}+1$ )

$$
\begin{aligned}
\hat{\mathbf{x}}(a) & =\left(\mathbf{F} \otimes \mathbf{I}_{N_{r}}\right) \hat{\mathbf{y}}(a) \\
& =\left(\mathbf{F} \otimes \mathbf{I}_{N_{r}}\right)\left(\mathbf{C}\left(\mathbf{F}^{-1} \otimes \mathbf{I}_{N_{t}}\right) \hat{\mathbf{s}}(a)+\hat{\mathbf{v}}(a)\right) \\
& =\hat{\mathbf{H}} \hat{\mathbf{s}}(a)+\hat{\mathbf{n}}(a)
\end{aligned}
$$

where $\hat{\mathbf{n}}(a)$ represents the frequency-domain noise, with i.i.d. zero-mean, complex Gaussian elements with variance $(1 / 2) \sigma_{n}^{2}$ per dimension, and $\hat{\mathbf{H}}$ is a block diagonal matrix, which is given by

$$
\hat{\mathbf{H}}=\left(\begin{array}{ccc}
\mathbf{H}(0) & & 0 \\
& \ddots & \\
0 & & \mathbf{H}\left(N_{c}-1\right)
\end{array}\right) .
$$

The $i$ th block diagonal element is the $N_{t} \times N_{r}$ MIMO channel of the $i$ th subcarrier and can be shown to be

$$
\mathbf{H}(i)=\sum_{l=0}^{L-1} \mathbf{G}(l) \exp \left(-j 2 \pi \frac{i l}{N_{c}}\right) .
$$

So, for this subcarrier, we may write

$$
\mathbf{x}(i, a)=\mathbf{H}(i) \mathbf{s}(i, a)+\mathbf{n}(i, a)
$$

which results in a flat-fading signal model per subcarrier. Note that if $N_{c}$ is a power of 2, the (I)DFT can be implemented efficiently with the (inverse) fast Fourier transform [I(FFT)].

\section{IMPLEMENTATION}

Synchronization is an essential task for any digital communication system and required for reliable reception of the transmitted data. From the perspective of physical layer design, proper synchronization algorithms are crucial to build a successful product. Therefore, for a potential application of MIMO to OFDM, it is important to examine the necessary changes to the preamble, time and frequency synchronization, channel estimation, synchronization tracking, and detection. Sections IV-A-F will give an overview of these necessary changes. In this evaluation, we will assume that the receiver gains (per branch) are already set by, e.g., an automatic gain control (AGC) algorithm. Since the AGC for a MIMO system does not differ significantly from the SISO case, it will not be treated in this paper.

\section{A. Preamble}

The radio communication system under investigation (i.e., WLAN) is a packet-switched system with a random access protocol. This essentially means that a receiver has no a priori knowledge about packet-arrival times. The random nature of the arrival times and the high data rates require the synchronization to be completed shortly after the start of the reception of a packet. To facilitate "quick" synchronization, the data packet is preceded with a known sequence (the preamble). The preamble is carefully designed to provide enough information for a good packet detection, frequency offset estimation, symbol timing, and channel estimation. With accurate knowledge of the MIMO channel elements, the MIMO processing can separate the signal components originating from the different transmit antennas.

To estimate the MIMO channel, it is important that the subchannels from the different TX antennas to every RX antenna can be uniquely identified. To achieve that, the preambles on the different TX antennas should be orthogonal and shift-orthogonal, for at least the channel length [14]. We have chosen for time orthogonality as shown in Fig. 3. 


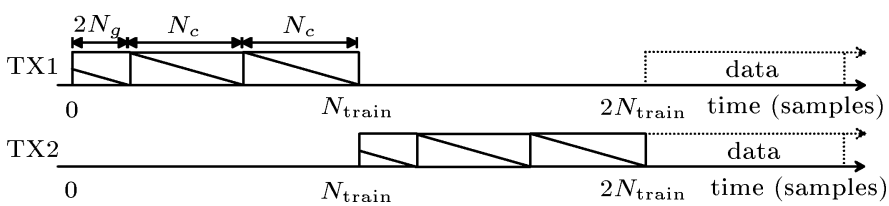

Fig. 3. Concept of a time orthogonal preamble for a MIMO configuration with two transmit antennas.

To perform frequency offset estimation, a periodicity in the preamble is desired since the phase rotation between time-delayed versions of the same symbol is a measure for the frequency offset [15]. Therefore, the proposed preamble consists of a concatenation of two identical training sequences per TX antenna.

Furthermore, to make the channel estimation less vulnerable to ISI, a CP of length $2 N_{g}$ is added. Altogether, this results in a preamble as depicted in Fig. 3 for a $2 \times 2$ system. Following the IEEE 802.11a standard, the training sequence is chosen to be the standards' long training symbol, with $N_{g}=16$ and $N_{c}=64$ [5], resulting in a nonzero part per TX antenna of $N_{\text {train }}=2 N_{g}+2 N_{c}$ samples. In the remainder of this paper, we will denote the signal on the $i$ th subcarrier of the long training symbol by $c(i)$.

Note that since the total length of the proposed preamble grows linearly with $N_{t}$, it is not highly efficient. This results in an increased overhead. More efficient channel training sequences for MIMO OFDM are proposed in [14]. It is claimed that training sequences for the different transmit antennas are optimal if they are orthogonal, as well as shift orthogonal over the sampling interval $\{-L+1, \ldots, L-1\}$. Since the impulse response length $L$ is not known in advance, the simplest implementation would be to choose a fixed orthogonality interval. If the shift orthogonality is not satisfied, however, training following the design rules of [14] becomes unstable, leading to an undesirable performance degradation. Therefore, stable but efficient channel estimation algorithms remain a topic for further research.

\section{B. Time Synchronization}

1) Frame Detection/Coarse Timing: The task of the frame detection (FD) is to identify the preamble in order to detect a packet arrival. This preamble detection algorithm can also be used as a coarse timing (CT) algorithm, since it inherently provides a rough estimate of the starting point of the packet. In literature different data-aided FD algorithms have been proposed for OFDM, [16]-[18]. A simple MIMO extension of Schmidl's algorithm [17] was proposed in [19]. All these algorithms are based on the correlation between the repeated symbols constituting the preamble.

We define the complex correlation $\Lambda$ between two subsequently received frames of $N_{c}$ samples on the $N_{r}$ receive antennas as

$$
\begin{aligned}
\Lambda(\tau) & =\sum_{n=\tau-\left(N_{c}-1\right)}^{\tau} \mathbf{r}^{H}\left(n-N_{c}\right) \mathbf{r}(n) \\
& =\sum_{n=\tau-\left(N_{c}-1\right)}^{\tau} \sum_{q=1}^{N_{r}} r_{q}{ }^{*}\left(n-N_{c}\right) r_{q}(n)
\end{aligned}
$$

where ${ }^{H}$ denotes the conjugate or Hermitian transpose, and $r_{q}(\tau)$ is the $n$th sample of the received signal on antenna $q$. The sum of the power of $N_{c}$ subsequently received samples on the $N_{r}$ receive antennas together, denoted by $P$, is defined as

$$
\begin{aligned}
P(\tau) & =\sum_{n=\tau-\left(N_{c}-1\right)}^{\tau} \mathbf{r}^{H}(n) \mathbf{r}(n) \\
& =\sum_{n=\tau-\left(N_{c}-1\right)}^{\tau} \sum_{q=1}^{N_{r}} r_{q}{ }^{*}(n) r_{q}(n) .
\end{aligned}
$$

Here, we propose to use the maximum-normalized-correlation (MNC) criterion for FD, which was proposed in [18] for a SISO OFDM system and shown to perform better than techniques proposed in [16] and [17]. In the MNC frame detection algorithm, the estimated start of the datapacket, i.e., the end of the preamble, is given by

$$
\tau_{\mathrm{FD}}=\arg \max _{\tau}\left[\frac{\sum_{p=1}^{N_{t}} 4\left|\Lambda\left(\tau_{p}\right)\right|^{2}}{\sum_{p=1}^{N_{t}} P\left(\tau_{p}-N_{c}\right)^{2}+P\left(\tau_{p}\right)^{2}}\right]
$$

where $\tau_{p}=\tau-\left(N_{t}-p\right) N_{\text {train }}$ to consider the offset of $N_{\text {train }}$ per TX branch (see Fig. 3). To avoid a false detection, i.e., a detection of a packet when none is present, a threshold should be set that triggers the above algorithm [20].

2) Symbol Timing: The symbol timing in an OFDM system decides where to place the start of the FFT window within the OFDM symbol. Although an OFDM system exhibits a guard interval (GI), making it somewhat robust against timing offsets, a nonoptimal symbol timing will cause more ISI and inter-carrier interference (ICI) in delay spread environments. This will result in a performance degradation.

The symbol timing proposed here is designed to minimize the amount of ISI and ICI that is generated in the system and is an extension of the technique proposed for SISO OFDM in [13, pp. 88-92]. Since it relies on the knowledge of the channel impulse responses (CIRs), their powers are estimated by correlating the received signals with the known training sequence

$$
\begin{aligned}
& \eta_{q}(\tau) \\
& =\left\|\sum_{k=0}^{N_{c}-1} r_{q}(\tau-k) \cdot \frac{1}{N_{c}} \sum_{i=0}^{N_{c}-1} c^{*}(i) e^{\left(-j 2 \pi i\left(N_{c}-1-k\right) / N_{c}\right)}\right\|^{2}
\end{aligned}
$$

Subsequently, the powers of the $N_{t} N_{r}$ impulse response estimates are summed. Note that the estimates of the powers of the CIRs, corresponding to the $N_{t}$ transmitters, are spaced $N_{\text {train }}$ samples apart in $\eta_{q}$ and that $\eta_{q}$ holds two estimates per TX spaced $N_{c}$ samples apart, caused by the repetition in the preamble. The sum of the powers is window integrated over the length of the GI of the OFDM symbol $N_{g}$. The joint symbol timing for the whole MIMO receiver $\tau_{\text {est }}$ is then found by 
searching the maximum of the window integral, i.e., a measure for the start of the payload:

$$
\tau_{\text {est }}=\arg \max _{\tau} \sum_{l=0}^{N_{g}-1} \sum_{p=1}^{N_{t}} \sum_{q=1}^{N_{r}} \sum_{n=0}^{1} \eta_{q}\left(\tau_{p}-l-n N_{c}\right) .
$$

This search window should be centered around $\tau=\tau_{\mathrm{FD}}$, as determined by (14).

\section{Frequency Synchronization}

The frequency synchronization has to correct for the frequency offset, which is caused by the difference in oscillator frequencies at the transmitter and the receiver. We estimate this frequency offset and compensate the received signals for it. The frequency offset can be estimated using the phase of the complex correlation $\Lambda$ between the two consecutive received training symbols, as was shown by Moose for a SISO OFDM system in [15] and further worked out in [17]. A simple MIMO extension of Moose's algorithm was proposed in [19]. Here, it is assumed that all transmit/receive branches of one MIMO transmitter/receiver use the same oscillator, which is a valid assumption if the different transmit/recieve branches are co-located. The best instant to estimate this frequency offset is at timing instant $\tau=\tau_{\mathrm{FD}}$. The estimated frequency offset $\Delta f_{\text {est }}$ is then given by

$$
\Delta f_{\text {est }}(\tau)=\frac{\theta_{\text {est }}(\tau)}{2 \pi T_{c}}=\frac{f_{s} \angle\left[\sum_{p=1}^{N_{t}} \Lambda\left(\tau_{p}\right)\right]}{2 \pi N_{c}}
$$

where $\theta_{\text {est }}$ denotes the phase of the summation of the complex correlations $\Lambda$ of the training symbols originating from the different transmitters, $f_{s}$ equals the sample frequency, $T_{c}=N_{c} / f_{s}$ is the training symbol duration and $\angle(\cdot)$ denotes the angle (in radians) of its argument. The maximum estimated frequency offset is limited, since the angle $\theta_{\text {est }}$ that can be estimated without phase ambiguity is limited to $\theta_{\max }= \pm \pi$. This relates to a maximum frequency offset of $\left|\Delta f_{\max }\right|=\left|\theta_{\max }\right| f_{s} / 2 \pi N_{c}=f_{s} / 2 N_{c}$, which equals half the subcarrier spacing. A larger range can be achieved by slightly changing the preamble, as was proposed in [17]. As an accuracy measure of the estimator, we consider the normalized variance of the estimator in an AWGN channel, as was derived in [15] for the SISO estimator. The normalized variance of the MIMO estimator is given by

$$
\begin{aligned}
\operatorname{var}\left(\frac{\Delta f_{\mathrm{est}}-\Delta f}{\Delta F}\right) & =E\left(\frac{\Delta f_{\mathrm{est}}-\Delta f}{\Delta F}\right)^{2} \\
& =\frac{1}{(2 \pi)^{2} N_{r} N_{c} \rho}
\end{aligned}
$$

where $\rho$ denotes the SNR per receive antenna, $\Delta f$ the actual frequency offset, and $\Delta F=f_{s} / N_{c}$ the subcarrier spacing. It can be shown that the variance of the estimator equals the mean squared error (MSE), since the estimator is unbiased. It is clear from (18) that the accuracy increases linearly with the number of receive antennas $N_{r}$. Note that the Cramér-Rao lower bound



Fig. 4. MSE of the frequency offset estimate for a $1 \times 1$ (solid lines) and a $3 \times 3$ (dashed lines) system from theory and simulations with AWGN and multipath channels.

is equal to this theoretical value of the variance, as was already concluded for the SISO version in [17].

In Fig. 4, the MSE of the frequency offset (FO) estimation is depicted as function of the average SNR per receive antenna. The theoretical value from (18) is shown together with results from Monte Carlo simulations averaged over 100000 channel realizations for exponentially decayed PDP having different values of rms delay spread (tds). Fig. 4 shows the results for the SISO and $3 \times 3$ configuration. It is clear that the theoretical value is a good estimate of the MSE for high SNR values but underestimates the MSE compared with simulation results for low SNR. In case of AWGN, we see an improvement of $4.8 \mathrm{~dB}$ in performance going from a $1 \times 1$ to a $3 \times 3$ configuration, as expected from (18).

The simulations with multipath channels show a degradation in performance with respect to the theoretical and the simulated AWGN case. The degradation is the worst for the flat-fading case and decreases when the rms delay spread increases. This is explained by the frequency diversity introduced by the delay spread channel. The degradation, however, is much smaller in the $3 \times 3$ case than in the SISO case. This shows that the frequency offset estimation in MIMO systems is more robust under different fading conditions, which can be explained by the space diversity introduced by the multiple antennas.

\section{Channel Estimation}

When time synchronization is performed at the receiver and after the received signals are corrected for the frequency offset, the channel can be estimated using the known training symbols within the preamble. When the timing is performed correctly, we know which received samples correspond to the training part. More precisely, we know exactly which part of the received preamble is sent by transmit antenna $p$ (see Fig. 3). Therefore, in the analysis of this subsection, we will omit the MIMO OFDM symbol index for brevity. Let us denote the Fourier transform 
of the symbol received on antenna $q$ that corresponds to the training symbol from transmitter $p$ by

$$
\hat{\mathbf{x}}_{q}^{p}=\left(\begin{array}{c}
x_{q}^{p}(0) \\
\vdots \\
x_{q}^{p}\left(N_{c}-1\right)
\end{array}\right) .
$$

Recall that $c(i)$ denotes the training signal on the $i$ th subcarrier. Then, rewriting the signal model of Section III, it can be shown that

$$
\hat{\mathbf{x}}_{q}^{p}=\mathbf{D}\left(\begin{array}{c}
h_{q p}(0) \\
\vdots \\
h_{q p}\left(N_{c}-1\right)
\end{array}\right)+\hat{\mathbf{n}}_{q}^{p}
$$

where $\mathbf{D}=\operatorname{diag}(\mathbf{c})$, with $\mathbf{c}=\left(c(0), \ldots, c\left(N_{c}-1\right)\right)^{T}$. Furthermore, $\hat{\mathbf{n}}_{q}^{p}$ is the frequency domain additive noise at the $q$ th receiver branch.

Now, the estimates of the channel coefficients can be obtained by

$$
\hat{\mathbf{h}}_{q p, \text { est }}=\left(\begin{array}{c}
h_{q p, \text { est }}(0) \\
\vdots \\
h_{q p, \text { est }}\left(N_{c}-1\right)
\end{array}\right)=\mathbf{D}^{\dagger} \hat{\mathbf{x}}_{q}^{p}
$$

where $\mathbf{D}^{\dagger}=\left(\mathbf{D}^{H} \mathbf{D}\right)^{-1} \mathbf{D}^{H}$ is the pseudo-inverse of $\mathbf{D}$. Since $\mathbf{D}$ is diagonal and the IEEE 802.11a training symbol has a constant amplitude of 1.0 over the (used) subcarriers [5], the processing of (21) can be simplified to

$$
\hat{\mathbf{h}}_{q p, \text { est }}=\mathbf{D}^{H} \hat{\mathbf{x}}_{q}^{p} .
$$

Note that because the proposed preamble contains a repetition of two identical training symbols, we can improve the accuracy of the channel estimate by averaging over the two corresponding received symbols before the above described processing is performed. Furthermore, note that performing the channel estimation in the time domain has a potential higher performance [21], but it is more sensitive to high timing offsets and high delay spreads due to extra constraints that are placed on the observation length, and therefore, the more robust implementation in frequency domain has been chosen.

\section{E. Synchronization Tracking Using Pilot Subcarriers}

As described in Sections IV-A-D, the processing of the preamble takes care of the initial synchronization of the MIMO OFDM receiver. It is, however, likely that the frequency offset will vary during the reception of the packet, making solely initial frequency synchronization insufficient. Furthermore, the system will experience phase noise (PN) invoked by the combination of RF oscillator and the phase-locked loop (PLL). From [22], it is clear that $\mathrm{PN}$ causes a common phase turn for all carriers, called common phase error (CPE), and a Gaussian-like ICI term. Since the CPE is equal for all subcarriers, it can be estimated and corrected for. An initial estimation is done inherently by the channel estimation. The CPE, however, changes on a symbol by symbol basis, making this initial correction insufficient.
It is, therefore, necessary to estimate and correct the rotation of the received constellation points caused by the FO and CPE on a symbol-by-symbol basis. A convenient method is to use pilot subcarriers, i.e., subcarriers containing known data, which are inserted into the data symbols. The rotation of these known pilot symbols, which are observed at the receiver, is a good measure for the CPE. This rotation for the ath symbol given by

$$
\vartheta(a)=\angle\left(\sum_{i \in \mathcal{P}} \sum_{p=1}^{N_{t}} \tilde{s_{p}}(i, a) s_{p}^{*}(i, a)\right)
$$

where $\mathcal{P}$ denotes the set of pilot subcarrier numbers, and $\tilde{\mathbf{s}}$ is the estimate, before slicing to the nearest constellation point, of the transmitted signal $\mathbf{s}$. This rotation can now be corrected for at the receiver by multiplying the complex values on all subcarriers of the $a$ th symbol with $\exp (-j \vartheta(a))$.

\section{F. MIMO Detection Algorithms}

Once a packet is detected and the synchronization and channel estimation are performed, the FFT begins retrieving the subcarrier signals. MIMO detection is applied to these signals on a subcarrier basis. In terms of spatial multiplexing, the MLD-based detection algorithm PAC SOMLD performs well. The disadvantage of this scheme is that its complexity grows exponentially with $N_{t}$ [23]. Therefore, we introduce another scheme, namely, PAC V-BLAST, whose performance is comparable, but whose complexity grows only polynomially with $N_{t}$ [23], [24] at the expense of latency.

1) Per-Antenna-Coded SOMLD: In case of per-antenna-coded soft-decision output MLD (PAC SOMLD), following [8], the detection and decoding block of Fig. 2 consists of $N_{c}$ maximum likelihood detectors that produce soft estimates (i.e., soft-decision output values) of the coded bits transmitted on the respective subcarrier. To find these soft-decision outputs, we can use the same approach as [25], where the log likelihood ratio (LLR) is used as an indication for the reliability of a bit. Suppose that at a given time instance, on a certain subcarrier, $K=N_{t} m$ bits are sent, where $m=\log _{2} M$ denotes the amount of bits used per $M$-QAM constellation point. Then, (omitting the subcarrier index), if $b_{k}$ is the $k$ th bit of the transmitted vector to estimate, the LLR for this bit is

$$
\operatorname{LLR}\left(b_{k}\right)=\ln \frac{\operatorname{Pr}\left(b_{k}=+1 \mid \mathbf{x}\right)}{\operatorname{Pr}\left(b_{k}=-1 \mid \mathbf{x}\right)}=\ln \frac{\sum_{\mathbf{s}_{j} \mid b_{k}=+1} \operatorname{Pr}\left(\mathbf{s}_{j} \mid \mathbf{x}\right)}{\sum_{\mathbf{s}_{j} \mid b_{k}=-1} \operatorname{Pr}\left(\mathbf{s}_{j} \mid \mathbf{x}\right)}
$$

where the ensemble $\mathbf{s}_{j}$ with $j=\{1, \ldots, J\}$ denotes all possible transmitted vectors at a given time instance on a certain subcarrier, and thus, $J=M^{N_{t}}$. When we apply Bayes' rule, the LLR becomes

$$
\operatorname{LLR}\left(b_{k}\right)=\ln \frac{\sum_{\mathbf{s}_{j} \mid b_{k}=+1} p\left(\mathbf{x} \mid \mathbf{s}_{j}\right) \operatorname{Pr}\left(\mathbf{s}_{j}\right)}{\sum_{\mathbf{s}_{j} \mid b_{k}=-1} p\left(\mathbf{x} \mid \mathbf{s}_{j}\right) \operatorname{Pr}\left(\mathbf{s}_{j}\right)} .
$$

Because the vectors $\mathbf{s}_{j}$ are equally likely to be transmitted, $\operatorname{Pr}\left(\mathbf{s}_{j}\right)$ is equal for all vectors $\mathbf{s}_{j}$. Since we assume that the vector $\mathbf{X}$ is the result of a MIMO transmission over a flat-fading 


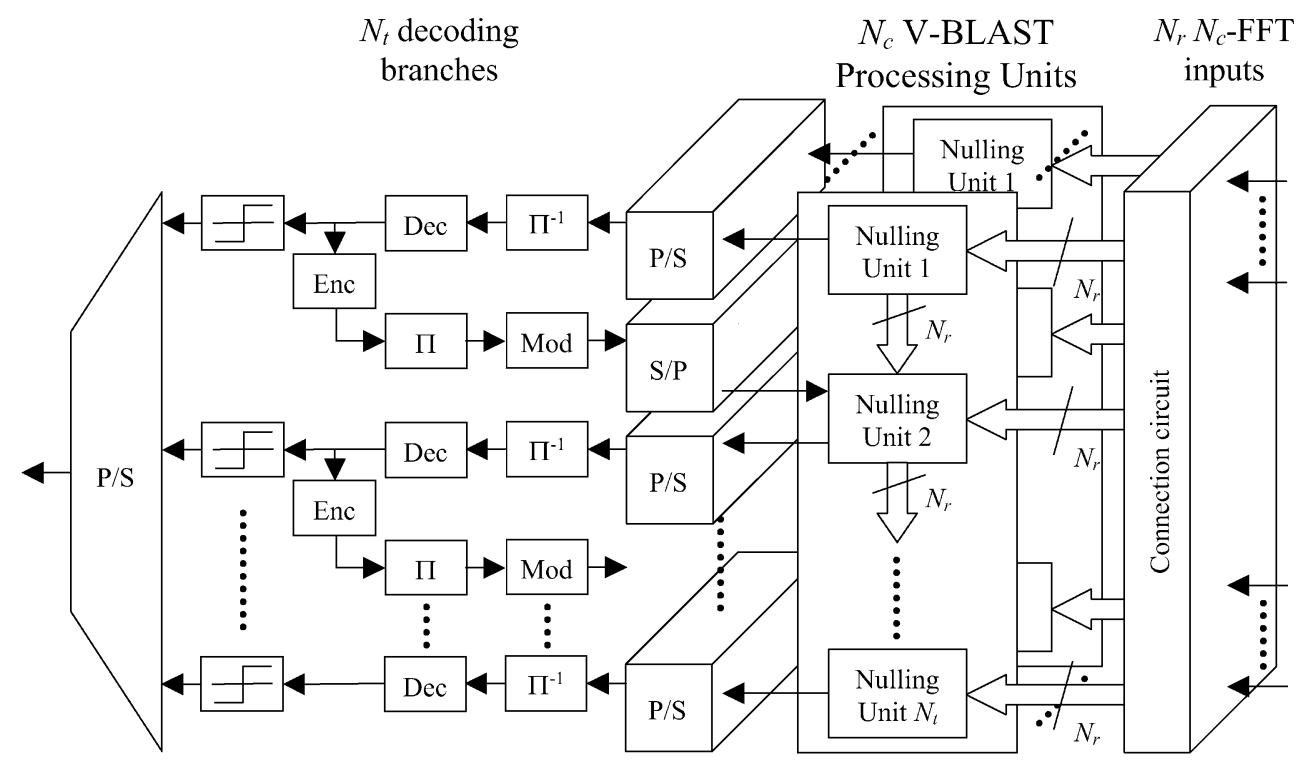

Fig. 5. PAC V-BLAST detection and decoding block.

Rayleigh channel (i.e., each subcarrier is treated flat over frequency), we know that this vector $\mathbf{x}$ has a complex multivariate normal distribution [8]. Therefore, for a given channel matrix $\mathbf{H}$, the conditional probability density function can be shown to be

$$
p\left(\mathbf{x} \mid \mathbf{H}, \mathbf{s}_{j}\right)=\frac{1}{\operatorname{det}(\pi \mathbf{Q})} \cdot e^{-\left(\mathbf{x}-\mathbf{H s}_{j}\right)^{H} \mathbf{Q}^{-1}\left(\mathbf{x}-\mathbf{H s}_{j}\right)}
$$

where $\mathbf{Q}$ is the covariance matrix and equals

$$
\begin{aligned}
\mathbf{Q} & =E\left[(\mathbf{x}-E[\mathbf{x}])(\mathbf{x}-E[\mathbf{x}])^{H}\right] \\
& =E\left[\left(\mathbf{x}-\mathbf{H} \mathbf{s}_{j}\right)\left(\mathbf{x}-\mathbf{H s}_{j}\right)^{H}\right] \\
& =E\left[\mathbf{n} \mathbf{n}^{H}\right]=\sigma_{n}^{2} \mathbf{I}_{N_{r}} .
\end{aligned}
$$

Finally, we obtain the LLR of $b_{k}$

$$
\operatorname{LLR}\left(b_{k}\right)=\ln \frac{\sum_{\mathbf{s}_{j} \mid b_{k}=+1} \exp \left(-\frac{D}{\sigma_{n}^{2}}\right)}{\sum_{\mathbf{s}_{j} \mid b_{k}=-1} \exp \left(-\frac{D}{\sigma_{n}^{2}}\right)}
$$

where $D=\left\|\mathbf{x}-\mathbf{H s}_{j}\right\|^{2}$. When applying the max-log approximation this results in

$$
\operatorname{LLR}\left(b_{k}\right) \approx \frac{1}{\sigma_{n}^{2}}\left(\min _{\mathbf{s}_{j} \mid b_{k}=-1} D-\min _{\mathbf{s}_{j} \mid b_{k}=+1} D\right) .
$$

Once the LLRs are computed, they are deinterleaved and decoded. Since the TX streams are encoded separately, the deinterleaving and decoding also has to be performed per stream. Hence, $N_{t}$ deinterleavers and Viterbi decoders are required to produce the final output.

Note that SOMLD involves an exhaustive search over all possible vectors $\mathbf{s}_{j}$, leading to a complexity that grows exponentially with $N_{t}$.

2) Per-Antenna-Coded V-BLAST: The fact that the complexity of MLD grows exponentially with the number of transmit antennas resulted in the search for less com- plex algorithms. One of the schemes that is based on minimum-mean-square-error (MMSE) detection with decision-feedback equalization (DFE) is called V-BLAST [24], [26]. The complexity of this scheme grows linearly with $N_{t}$ [24], and when using the proposed optimal successive interference cancellation (OSIC) in [26], the performance degradation with respect to MLD is small compared with linear techniques like zero-forcing [4]. In this paper, we propose an extension to V-BLAST, making use of PAC at the transmitter. The idea is to first go through the decoding stage before the successive interference cancellation (SIC) is executed. In this way, forward error correcting (FEC) coding is performed on the SIC information. How this can be applied to MIMO OFDM is schematically represented in Fig. 5.

To improve the performance even more, the V-BLAST detection should produce soft-decision outputs. In order to obtain them, the estimated values of each nulling unit should not be sliced to their respective QAM points, as done in [26], but they should go through a SISO implementation, i.e., the $1 \times 1$ version of SOMLD described above.

Note that since the SIC information is passed through a decoder and encoder stage, a disadvantage of this scheme could be its latency, but when the interleaver is small, e.g., equivalent to the size of the interleaver defined in the IEEE 802.11a standard [5], and convolutional encoding and Viterbi decoding is used, the encoder in the SIC feedback loop can start its operation as soon as the Viterbi decoder produces outputs. Then, for a limited number of transmit antennas, the latency is manageable.

\section{PERFORMANCE}

\section{A. Simulation Results}

This subsection shows the results of a number of simulations that are performed to compare the BER performance of the two PAC detection schemes described in Section IV. The main simulation parameters are based on the IEEE 802.11a standard and summarized in Table I. 
TABLE I

SimULATION PARAMETERS, BASED ON THE 802.11A OFDM STANDARD

\begin{tabular}{c|c}
\hline System Parameter & Parameter Value \\
\hline Modulation & Binary Phase Shift Keying \\
Bandwidth & $20 \mathrm{MHz}$ \\
Number of subcarriers $N_{c}$ & 64 \\
Number of data subcarriers & 48 \\
Number of pilot tones & 4 \\
OFDM Symbol duration & $4 \mu \mathrm{s}$ \\
Guard Interval & $800 \mathrm{~ns}$ \\
\hline
\end{tabular}

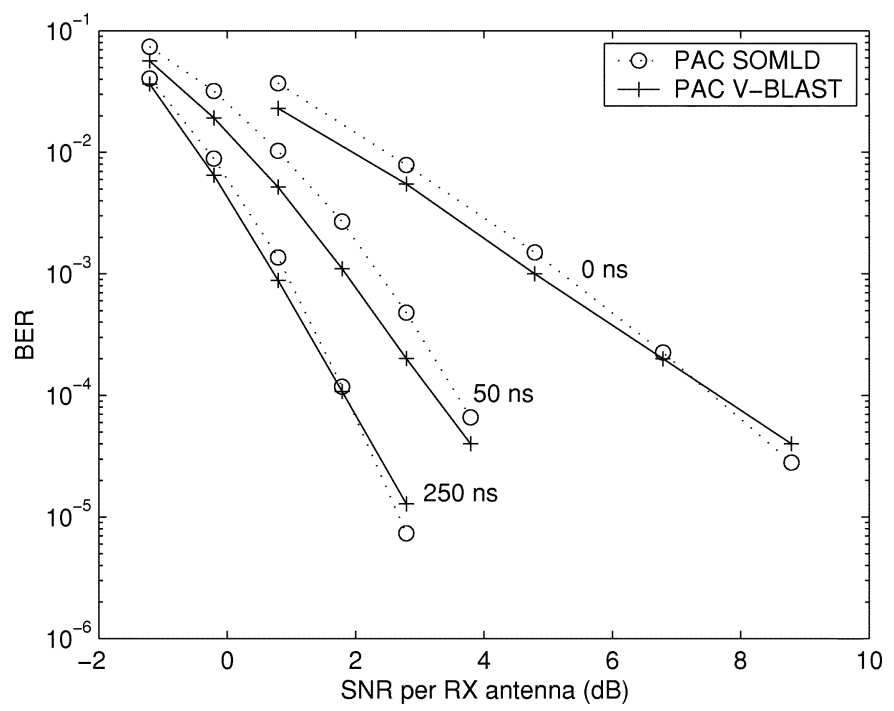

Fig. 6. BER performance for $4 \times 4$ PAC schemes, BPSK, rate $1 / 2$ coding, and frequency-selective Rayleigh fading with an exponential decaying PDP with different rms delay spreads.

The BER performance is obtained by averaging over 50000 108 byte packets. Assuming quasistatic fading, every packet is sent through a channel realization, of which the elements $g_{q p}(l)$ are modeled according to Section III with an exponentially decaying PDP (i.e., the ensemble $P(l)$ falls off exponentially) with an rms delay spread of 0,50 , and $250 \mathrm{~ns}$. In order to correct for subcarriers in deep fades, a FEC code across the subcarriers is used: rate $1 / 2$ convolutional coding with constraint length 7 and generator polynomials (133 171). Furthermore, the IEEE 802.11a interleaver is used [5].

With these parameters, the BER performance versus the SNR per receive antenna is depicted in Fig. 6, for PAC SOMLD and V-BLAST, for different delay spreads and for a $4 \times 4$ antenna setup. Note that in obtaining these results, perfect knowledge of the channel, i.e., perfect channel state information (CSI), and perfect synchronization is assumed at the receiver.

From the results of Fig. 6, we see that for low SNR values, the performance of PAC V-BLAST is better than that of PAC SOMLD, but for high SNRs, the performance of PAC V-BLAST is worse. The latter can be explained by the fact that the diversity order of V-BLAST for high SNRs tends toward $N_{r}-N_{t}+1$, whereas that of SOMLD is equal to $N_{r}$ [4]. The former can be explained by the way the soft-decision output values are

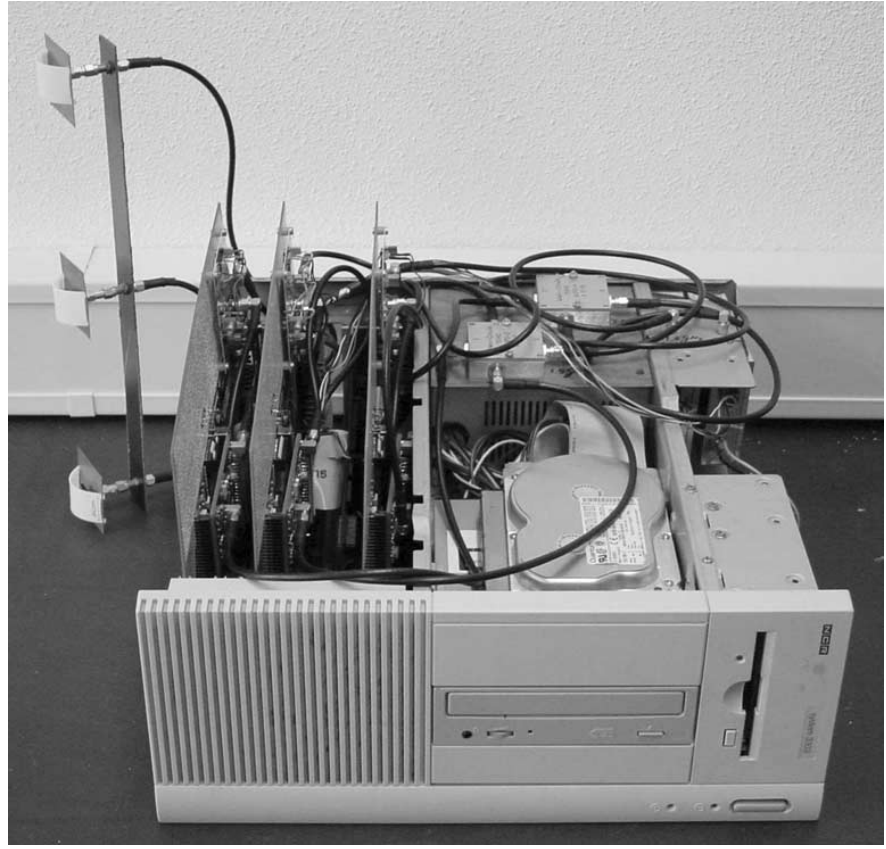

Fig. 7. Receiver equipment: PC with three receiver boards (i.e., the three receiver branches).

generated. Due to nonorthogonal channels, received MIMO vectors will have dependent elements, which again will result in dependent soft-values for PAC SOMLD, as defined by (24). It is well known that the Viterbi decoder only performs optimally if the input values are independent. A solution would be to calculate joint LLRs [27] and change the decoder to handle these joint soft-values accordingly. Since V-BLAST is based on the MMSE algorithm, it first orthogonalizes the data streams and then determines the soft values, and hence, V-BLAST does not have the above-mentioned problem, which explains its better performance.

\section{B. Measurement Setup}

A MIMO test system has been built with the goal to verify the theoretical MIMO propagation studies and to serve as a platform for MIMO algorithm development. The test system operates in the 5. $\times \mathrm{GHz}$ ISM band and is capable of transceiving broadband signals with a bandwidth up to $20 \mathrm{MHz}$.

The digital processing is kept offline, allowing for quick exploration of different MIMO algorithms. The testbed is built up with in-house developed components. To access the hardware, two PC's are used: the transmitter (TX) and receiver (RX) platform, respectively. Each PC contains three boards, where each single board consists of one entire transmitter or receiver branch, resulting in a $3 \times 3$ MIMO system. Each board consists of a baseband part, an IF part, and a RF front-end based on a $5 . \times \mathrm{GHzGaAs}$ radio chip. A picture of the receiver equipment is given in Fig. 7.

The TX baseband part is set up to send signals at zero-IF, whereas the RX downconverts the received signals to a low-IF frequency. In the baseband processing, this low-IF signal is down converted digitally, using the so-called sampled-IF principle. This setup has been chosen to be able to easily filter out the dc offset and to easily calibrate the $I-Q$ imbalance since its main source is the transmitter. 


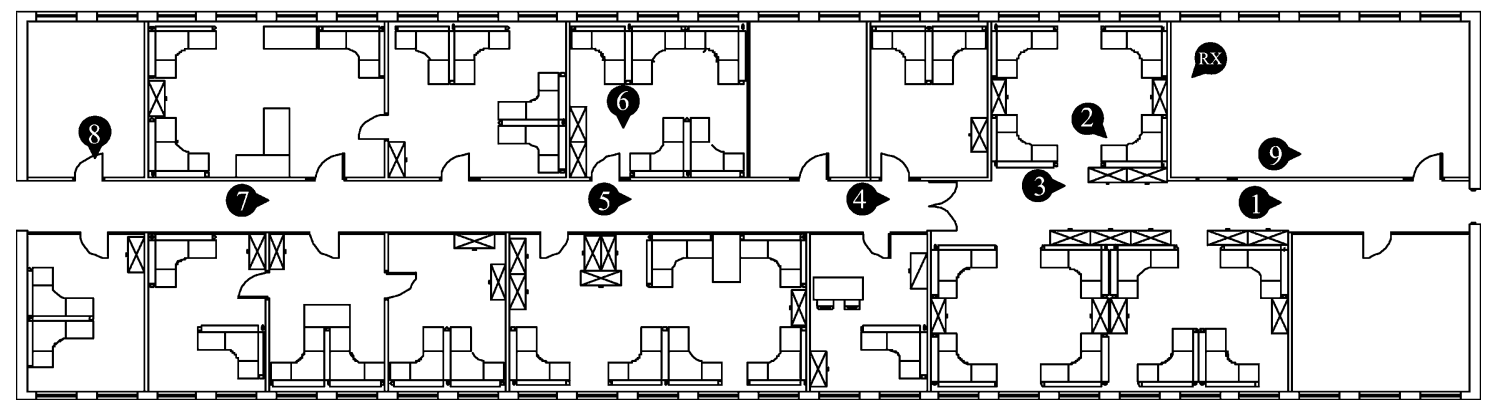

Fig. 8. Floor plan of the office where the measurements were performed $(42 \mathrm{~m} \times 12.7 \mathrm{~m})$, including the RX and TX locations and orientations.

The baseband processing is built around two field programmable gate arrays (FPGAs) per board. In our setup, the FPGAs are mainly configured as memory banks. At the transmitter, waveforms are loaded into and sent from the memory banks, and they are recorded at the receiver. These recorded data are processed offline with software written in MATLAB to, e.g., determine the performance by means of bit errors. By transmitting multiple packets, BER tests can be performed in order to evaluate different MIMO algorithms in real-life communication channels.

\section{Comparison}

Measurements with the above described test system were done in a wing on the third floor of the Agere Systems building in Nieuwegein, The Netherlands, which can be regarded as a typical office environment. The wing is $42 \mathrm{~m} \times 12.7 \mathrm{~m}$, and its floor plan is shown in Fig. 8. The inner walls of the wing are modular walls, whereas the rest of the walls and floors consists of concrete. Besides the walls, the desks and metal cupboards are depicted. Furthermore, the RX and nine different TX positions and orientations that were used for the measurements are shown. The applied antennas were omnidirectional, but due to obstruction by the test system, their effective patterns covered only a half plane. The antenna spacing was fixed at two wavelengths. Each transmit antenna transmitted at an average power of $50 \mathrm{~mW}$. The average SNR per RX antenna and the rms delay spread that we measured per location are given in Table II. These values were measured at baseband level and, thus, include system influences. This means that the estimated rms delay spread includes the influence of filters at both transmitter and receiver. The memory of the transmitter has a storage capacity of 19 MIMO OFDM data symbols, which we always fully exploited, leading to different packet lengths for different data rates. The tested data rates and corresponding packet lengths, i.e., the number of information bits, are given in Table III. To obtain the average performance, 1000 packets were transmitted per rate. The BER and PER performance for different locations and different rates are depicted in Figs. 9-11. Note that for certain rates, no performance value is given; this corresponds to the fact that at these rates, all 1000 packets were received correctly. Furthermore, note that the $1 \times 1$ measurements were only performed for locations 5,6 , and 7 and were obtained with the same test system by switching off two of the three boards at both TX and RX.

In Figs. 9-11, it can be observed that the performance of the $3 \times 3$ measurements is worse than the performance of its
TABLE II

Average SNR PER RX ANTENNA AND RMs Delay SpREad Versus Position

\begin{tabular}{c|c|c}
\hline Position & $\begin{array}{c}\text { Average SNR } \\
(\mathrm{dB})\end{array}$ & $\begin{array}{c}\text { rms delay spread } \\
(\mathrm{ns})\end{array}$ \\
\hline 1 & 24 & 98 \\
2 & 24 & 97 \\
3 & 24 & 100 \\
4 & 23 & 104 \\
5 & 25 & 91 \\
6 & 24 & 100 \\
7 & 22 & 109 \\
8 & 14 & 131 \\
9 & 26 & 100 \\
\hline
\end{tabular}

TABLE III

DATA RATES AND PACKET LENGTHS

\begin{tabular}{c|c|c|c|c}
\hline $\begin{array}{c}\text { Throughput } \\
(\mathrm{Mb} / \mathrm{s})\end{array}$ & $\begin{array}{c}\text { Antenna } \\
\text { setup }\end{array}$ & Modulation & $\begin{array}{c}\text { Coding } \\
\text { Rate }\end{array}$ & $\begin{array}{c}\text { Packetlength } \\
\text { (Bytes) }\end{array}$ \\
\hline 6 & $1 \times 1$ & BPSK & $1 / 2$ & 57 \\
9 & $1 \times 1$ & BPSK & $3 / 4$ & 85.5 \\
12 & $1 \times 1$ & QPSK & $1 / 2$ & 114 \\
18 & $1 \times 1$ & QPSK & $3 / 4$ & 171 \\
24 & $1 \times 1$ & 16-QAM & $1 / 2$ & 228 \\
36 & $1 \times 1$ & $16-$ QAM & $3 / 4$ & 342 \\
48 & $1 \times 1$ & $64-\mathrm{QAM}$ & $2 / 3$ & 456 \\
54 & $1 \times 1$ & $64-\mathrm{QAM}$ & $3 / 4$ & 513 \\
18 & $3 \times 3$ & BPSK & $1 / 2$ & 171 \\
27 & $3 \times 3$ & BPSK & $3 / 4$ & 256.5 \\
36 & $3 \times 3$ & QPSK & $1 / 2$ & 342 \\
54 & $3 \times 3$ & QPSK & $3 / 4$ & 513 \\
72 & $3 \times 3$ & 16-QAM & $1 / 2$ & 684 \\
108 & $3 \times 3$ & 16-QAM & $3 / 4$ & 1026 \\
144 & $3 \times 3$ & $64-\mathrm{QAM}$ & $2 / 3$ & 1368 \\
162 & $3 \times 3$ & 64-QAM & $3 / 4$ & 1539 \\
\hline
\end{tabular}

$1 \times 1$ counterpart with one third of the data rate. To have a PER of $1 \%$, it can be shown that in the $1 \times 1$ case, a rate of 54, 36, and $24 \mathrm{Mb} / \mathrm{s}$ can be achieved for locations 5, 6, and 7 , respectively. For the $3 \times 3$ case, these rates are, respectively, 108,54 , and $54 \mathrm{Mb} / \mathrm{s}$, resulting in an average throughput enhancement of 1.92. For well-conditioned MIMO channels, i.e., 

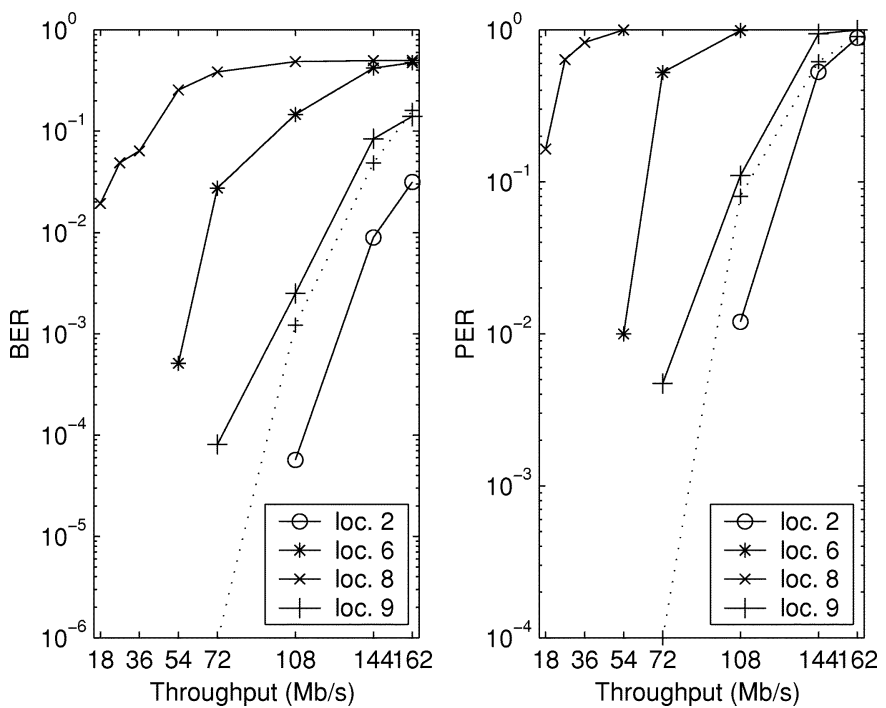

Fig. 9. Measurement results of a $3 \times 3$ system with PAC V-BLAST detection for the TX locations 2, 6, 8, and 9. For location 9, the measurements are compared with simulations (dotted line), with an average SNR per receive antenna of $26 \mathrm{~dB}$ and a rms delay spread of $100 \mathrm{~ns}$.
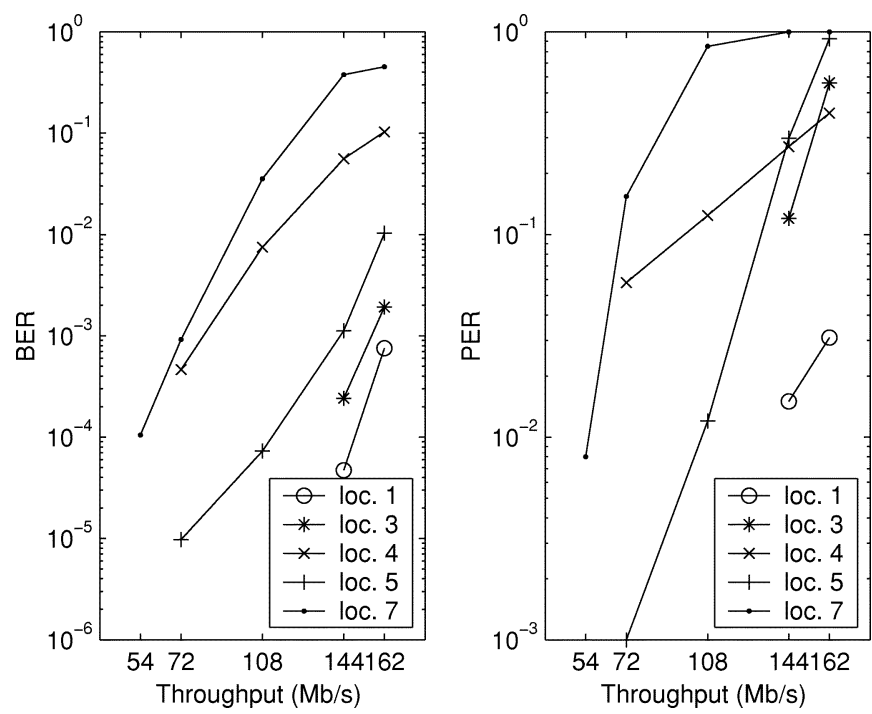

Fig. 10. Measurement results of a $3 \times 3$ system with PAC V-BLAST detection for the TX locations $1,3,4,5$, and 7 .

channels with i.i.d. channel elements, the throughput could theoretically be improved by a factor of 3 . Clearly, the measured MIMO channels do not provide this improvement. Two arguments can explain this fact: First, the environment does not provide enough scattering, leading to ill-conditioned MIMO channels, and second, mutual coupling between the branches at the transmitter and the receiver leads to performance degradation. Since our system is not shielded very well, as can be seen from Fig. 7, the last point most likely results in the highest performance loss in our case.

Next to measurements, simulations were performed to evaluate the packet error rate (PER) performance for the $3 \times 3$ rates $72,108,144$, and $162 \mathrm{Mb} / \mathrm{s}$ (see Table III) and to compare the results with the measurements results. Location 9 was used for this comparison (see Fig. 8). At this position, we observed an rms delay spread of about $100 \mathrm{~ns}$ and an average SNR per RX
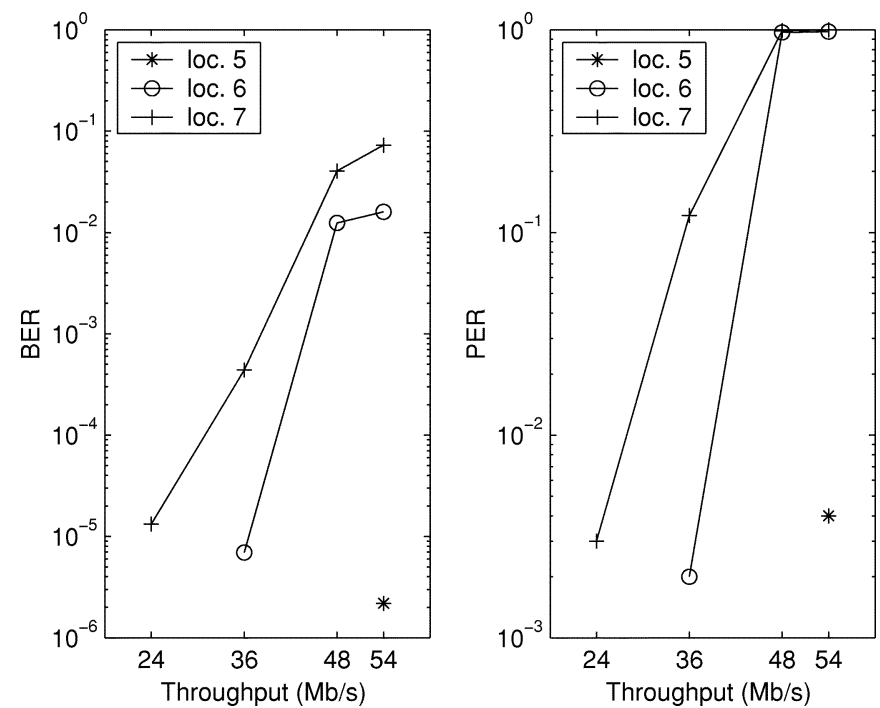

Fig. 11. Measurement results of a $1 \times 1$ system with PAC V-BLAST detection for the TX locations 5,6 , and 7 .



Fig. 12. PER simulation results of PAC V-BLAST for a $1 \times 1$ and $3 \times 3$ configuration, with perfect CSI at the receiver (dotted line) and with channel estimation (solid lines), different data rates, and frequency-selective Rayleigh fading with an exponential decaying PDP with $100 \mathrm{~ns}$ rms delay spread.

antenna of $26 \mathrm{~dB}$. These parameters were used in the simulations. Furthermore, perfect synchronization and i.i.d. channel elements were assumed, and the PER was obtained by averaging over 10000 packets. The results are shown in Fig. 12. All but one curve are obtained by performing channel estimation, and the other curve (based on 16-QAM modulation and coding rate $1 / 2$ ) is generated assuming perfect CSI at the receiver.

From these simulations results, we see that the performance deteriorates going to higher data rates, and we observe that applying channel estimation results in a loss of more than $4 \mathrm{~dB}$. A more advanced channel estimation algorithm might reduce this loss (see Sections IV-A and D ). Furthermore, we note that the $3 \times 3$ curves fall off faster than the $1 \times 1$ curve $(54 \mathrm{Mb} / \mathrm{s}$, 64-QAM, rate 3/4 and a packet length of 513 bytes), such that at high SNRs, most of the higher MIMO rates outperform the 
SISO $54 \mathrm{Mb} / \mathrm{s}$ rate, even having longer packets. This can be explained by the fact that the MIMO configurations benefit not only from the frequency diversity but from the spatial diversity as well.

Taking the simulation results at an SNR of $26 \mathrm{~dB}$, we can evaluate the performance of the measurements with the test system for location 9. For different data rates and a rms delay spread of $100 \mathrm{~ns}$, the results are depicted in Fig. 9 by the dotted lines. We clearly see that the performance of the test system in a real environment is worse than the performance of the idealized simulations. This can most likely be explained by system degradations that are not taken into account in the simulations, such as mutual coupling, residual $I-Q$ imbalance, errors in the frequency offset estimation, phase noise, and quantization. Another explanation could be the assumption of i.i.d. channel elements in the simulations.

\section{CONCLUSIONS}

An overview is given of the (changes in) signal processing, which is required to extend the physical layer of an OFDM system to multiple-input multiple-output (MIMO) OFDM. The signal processing of frame detection, time synchronization, frequency synchronization, channel estimation, synchronization tracking using pilot subcarriers, and MIMO detection algorithms is discussed. As a test case, the MIMO extension of the OFDM standard IEEE 802.11a is considered, but the results can be applied more generally. The necessary processing is not only evaluated through simulations but also by measurements using a $3 \times 3$ MIMO test system set up in a typical office environment.

From simulations, it is concluded that the MIMO detection scheme per-antenna-coding (PAC) V-BLAST, for low SNRs, outperforms the more complex PAC soft output MLD. Moreover, the $3 \times 3$ PAC V-BLAST achieves a comparable performance as the $1 \times 1$ variant, even though the throughput is three times higher. Furthermore, the measurements performed with the test system [with a (partly offline) implementation of the complete signal processing] in a practical environment showed a slightly worse performance than the idealized simulation results. The explanation is that in the simulations, system degradations such as phase noise and quantization as well as propagation effects such as ill-conditioned MIMO channels are not taken into account.

Finally, measurements show that an implementation of a $3 \times 3$ MIMO OFDM system achieves about a two times higher throughput than its $1 \times 1$ counterpart at a given range. Two reasons can be found for not reaching the theoretical tripling of the throughput: first, mutual coupling between the branches at the transmitter and receiver side and, second, the maximum throughput enhancement by a factor of 3 can only be achieved in well-conditioned MIMO channels, i.e., having i.i.d. channel elements.

\section{ACKNOWLEDGMENT}

The authors would like to acknowledge the suggestions of J. Hammerschmidt, I. Modonesi, and X.-J. Tao, and the anonymous reviewers. Furthermore, R. van der Burg, G. Draijer, and
R. van Poppel also deserve recognition for their work on the implementation and calibration of the MIMO test system.

\section{REFERENCES}

[1] G. J. Foschini and M. J. Gans, "On limits of wireless communications in a fading environment when using multiple antennas," Wireless Pers. Commun., vol. 6, no. 3, pp. 311-335, Mar. 1998.

[2] G. G. Raleigh and J. M. Cioffi, "Spatio-temporal coding for wireless communication," IEEE Trans. Commun., vol. 46, pp. 357-366, Mar. 1998.

[3] V. Tarokh, N. Seshadri, and A. R. Calderbank, "Space-time codes for high data rate wireless communication: Performance criterion and code construction," IEEE Trans. Inform Theory, vol. 44, pp. 744-756, Mar. 1998.

[4] A. van Zelst, "Space division multiplexing algorithms," in Proc. 10th Mediterranean Electrotech. Conf., vol. 3, 2000, pp. 1218-1221.

[5] IEEE 802.11a Stand., ISO/IEC 8802-11:1999/Amd 1:2000(E).

[6] Further Higher-Speed Physical Layer Extension in the $2.4 \mathrm{GHz}$ Band, Draft IEEE 802.11g Stand..

[7] R. van Nee, G. Awater, M. Morikura, H. Takanashi, M. Webster, and K. Helford, "New high rate wireless LAN standards," IEEE Commun. Mag., vol. 37, pp. 82-88, Dec. 1999.

[8] A. van Zelst, R. van Nee, and G. A. Awater, "Space division multiplexing (SDM) for OFDM systems," in Proc. IEEE Veh. Technol. Conf., May 2000, pp. 1070-1074.

[9] R. J. Piechocki, P. N. Fletcher, A. R. Nix, C. N. Canagarajah, and J. P. McGeehan, "Performance evaluation of BLAST-OFDM enhanced hiperlan/2 using simulated and measured channel data," Electron. Lett., vol. 37, no. 18, pp. 1137-1139, Aug. 2001.

[10] M. D. Batariere, J. F. Kepler, T. P. Krauss, S. Mukthavaram, J. W. Porter, and F. W. Vook, "An experimental OFDM system for broadband mobile communications," in Proc. IEEE Veh. Technol. Conf., vol. 4, 2001, pp. 1947-1951.

[11] H. Sampath, S. Talwar, J. Tellado, V. Erceg, and A. Paulraj, "A fourthgeneration MIMO-OFDM: Broadband wireless system: Design, performance, and field trial results," IEEE Commun. Mag., vol. 40, no. 9, pp. 143-149, Sept. 2002.

[12] X. Li, H. Huang, G. J. Foschini, and R. A. Valenzuela, "Effects of iterative detection and decoding on the performance of BLAST," in Proc. IEEE Global Telecommun. Conf., vol. 2, pp. 1061-1066.

[13] R. van Nee and R. Prasard, OFDM for Wireless Multimedia Communications. Norwell, MA: Artech House, 2000.

[14] I. Barhumi, G. Leus, and M. Moonen, "Optimal training sequences for channel estimation in MIMO OFDM systems in mobile wireless channels," in Proc. Int. Zurich Seminar Broadband Commun., Zurich, Switzerland, Feb. 2002, pp. 44-1-44-6.

[15] P. H. Moose, "A technique for orthogonal frequency division multiplexing frequency offset correction," IEEE Trans. Commun., vol. 42, pp. 2908-2914, Oct. 1994

[16] T. Keller and L. Hanzo, "Orthogonal frequency division multiplex synchronization techniques for wireless local area networks," in Proc. PIMRC, Taipei, Taiwan R.O.C., 1996, pp. 963-967.

[17] T. M. Schmidl and D. C. Cox, "Robust frequency and timing synchronization for OFDM," IEEE Trans. Commun., vol. 45, pp. 1613-1621, Dec. 1997.

[18] S. H. Müller-Weinfurtner. On the optimality of metrics for coarse frame synchronization in OFDM: A comparison. presented at Proc. PIMRC [Online]. Available: http://www.uwsmw.de/pub_stefan.html

[19] A. N. Mody and G. L. Stüber, "Synchronization for MIMO OFDM systems," in Proc. IEEE Global Commun. Conf., vol. 1, Nov. 2001, pp. 509-513.

[20] M. Lasanen, J. Rautio, and M. Nissillä, "Timing synchronization of the WIND-FLEX OFDM prototype," in Proc. IST Mobile Wireless Telecommun. Summit, June 17-19, 2002.

[21] J. Heiskala and J. Terry, OFDM Wireless LANs: A Theoretical and Practical Guide. Indianapolis, IN: Sams, 2002.

[22] A. G. Armada and M. Calvo, "Phase noise and subcarrier spacing effects on the performance of an OFDM communication system," IEEE Commun. Lett., vol. 2, no. 1, pp. 11-13, Jan. 1998.

[23] A. van Zelst, "Per-antenna-coded schemes for MIMO OFDM," in Proc. IEEE Int. Conf. Commun., vol. 4, Anchorage, AK, May 2003, pp. 2832-2836.

[24] B. Hassibi, "An efficient square-root algorithm for BLAST,", http://mars.bell-labs.com/cm/ms/what/mars/index.html. 
[25] J. Hagenauer and P. Hoeher, "A viterbi algorithm with soft-decision outputs and its applications," in Proc. IEEE Global Telecommun. Conf., Dallas, TX, Nov. 1989, pp. 47.1.1-47.1.7.

[26] P. W. Wolniansky, G. J. Foschini, G. D. Golden, and R. A. Valenzuela, "V-BLAST: An architecture for realizing very high data rates over the rich-scattering wireless channel," in Proc. URSI Int. Symp. Signals, Syst., Electron., pp. 295-300.

[27] H.-J. Su and E. Geraniotis, "Space-time turbo codes with full antenna diversity," IEEE Trans. Commun., vol. 49, pp. 47-57, Jan. 2001.

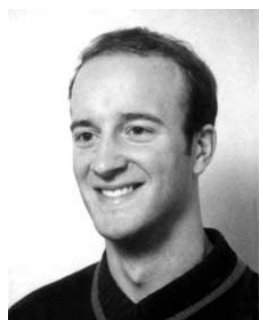

Allert van Zelst (S'96) was born in Waalwijk, The Netherlands, in 1976. He received the M.Sc. degree in electrical engineering from the Eindhoven University of Technology (TU/e), Eindhoven, The Netherlands, in 1999. Currently, he is pursuing the Ph.D. degree at the radio communications chair of the TU/e.

His general research interests include signal processing for broadband wireless communication systems, multiple antenna techniques, and wireless channel modeling.

Mr. van Zelst won the second prize of the IEEE Region 8 Student Paper Contest 2000 for a paper entitled "Space Division Multiplexing Algorithms." He is a member of the Dutch Electronics and Radio Society (NERG)

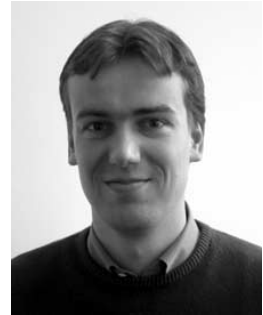

Tim C. W. Schenk (S'01) was born in Heerle, The Netherlands, in 1978. He received the M.Sc. degree in electrical engineering from Eindhoven University of Technology, Eindhoven, The Netherlands, in 2002, where he is currently pursuing the Ph.D. degree.

His research interests include signal processing for broadband multiple antenna systems and wireless channel modeling.

Mr. Schenk is a member of the Royal Institute of Engineers (KIvI), The Netherlands, and the Dutch Electronics and Radio Society (NERG). 